\title{
Identification of slow magnetosonic wave trains and their evolution in 3-D compressible turbulence simulation
}

\author{
L. Zhang ${ }^{1}$, L.-P. Yang ${ }^{2,1}$, J.-S. He ${ }^{1}$, C.-Y. Tu ${ }^{1}$, L.-H. Wang ${ }^{1}$, E. Marsch ${ }^{3}$, and X.-S. Feng ${ }^{2}$ \\ ${ }^{1}$ School of Earth and Space Sciences, Peking University, 100871 Beijing, China \\ ${ }^{2}$ SIGMA Weather Group, State Key Laboratory for Space Weather, Center for Space Science and Applied Research, Chinese \\ Academy of Sciences, Beijing, China \\ ${ }^{3}$ Institute for Experimental and Applied Physics, Christian-Albrechts-Universität zu Kiel, Kiel, Germany
}

Correspondence to: J.-S. He (jshept@pku.edu.cn)

Received: 13 August 2014 - Revised: 20 November 2014 - Accepted: 20 November 2014 - Published: 7 January 2015

\begin{abstract}
In solar wind, dissipation of slow-mode magnetosonic waves may play a significant role in heating the solar wind, and these modes contribute essentially to the solar wind compressible turbulence. Most previous identifications of slow waves utilized the characteristic negative correlation between $\delta|B|$ and $\delta \rho$. However, that criterion does not well identify quasi-parallel slow waves, for which $\delta|B|$ is negligible compared to $\delta \rho$. Here we present a new method of identification, which will be used in 3-D compressible simulation. It is based on two criteria: (1) that $V_{\mathrm{p}} \boldsymbol{B}_{0}$ (phase speed projected along $\boldsymbol{B}_{0}$ ) is around $\pm c_{\mathrm{s}}$, and that (2) there exists a clear correlation of $\delta v_{\|}$and $\delta \rho$. Our research demonstrates that if $v_{\mathrm{A}}>c_{\mathrm{s}}$, slow waves possess correlation between $\delta v_{\|}$ and $\delta \rho$, with $\delta \rho / \delta v_{\|} \approx \pm \rho_{0} / c_{\mathrm{s}}$. This method helps us to distinguish slow-mode waves from fast and Alfvén waves, both of which do not have this polarity relation. The criteria are insensitive to the propagation angle $\theta_{\boldsymbol{k} \boldsymbol{B}}$, defined as the angle between wave vector $\boldsymbol{k}$ and $\boldsymbol{B}_{0}$; they can be applied with a wide range of $\beta$ if only $v_{\mathrm{A}}>c_{\mathrm{S}}$. In our numerical simulation, we have identified four cases of slow wave trains with this method. The slow wave trains seem to deform, probably caused by interaction with other waves; as a result, fast or Alfvén waves may be produced during the interaction and seem to propagate bidirectionally away. Our identification and analysis of the wave trains provide useful methods for investigations of compressible turbulence in the solar wind or in similar environments, and will thus deepen understandings of slow waves in the turbulence.
\end{abstract}

Keywords. Interplanetary physics (MHD waves and turbulence)

\section{Introduction}

The turbulent solar wind contains various magnetohydrodynamic (MHD) and kinetic waves (Tu and Marsch, 1995; Goldstein et al., 1995; Bruno and Carbone, 2005; Marsch, 2006; He et al., 2011; Podesta and Gary, 2011; He et al., 2012; Salem et al., 2012). Alfvén waves hardly dissipate, and therefore this wave mode is observed ubiquitously (Belcher and Davis, 1971; Wang et al., 2012). Fast magnetosonic waves are compressible and are thus easily damped for a plasma $\beta$ of order unity. Moreover, their energy propagates in divergent directions from the source, and therefore their wave energy dilutes by spreading all around, making fast waves more difficult to observe in the solar wind (Tu and Marsch, 1995). Though slow waves are compressible and thought to be prone to damping, in the solar wind they tend to possess $k_{\|}$small enough to avoid strong damping (Chen et al., 2012). Moreover, their wave energy is mainly confined within magnetic flux tubes and transfers approximately along the magnetic field. Sequentially it is more likely to observe slow waves. Kellogg and Horbury (2005) researched density oscillations and found a compressible mode of waves that may be interpreted as slow waves. Howes et al. (2012) found negative correlations of $\delta n$ and $\delta B_{\|}$in data from the Wind spacecraft, and suggested that the principal compressible component of inertial range turbulence is the kinetic slow mode. Yao et al. (2013) (Y13) reported MHD slow waves observed with the Wind spacecraft. Their study described an event with slow waves embedded in Alfvén fluctuations, where the plasma $\beta \approx 1$. These cases were identified using the criteria given by Gary and Winske (1992), i.e., compressibility $C_{p}$ 
and dimensionless cross-helicity $\sigma_{\mathrm{c}}$. However, these criteria require a wave train with a pure mode, and may fail when waves of different modes mix up. Though cases reported by Y13 did agree well with this requirement, the usability of these criteria is limited. (Various linear spectral models (e.g., Klein et al., 2012) can be used for the problem.)

Recently simulations of these wave modes in MHD turbulence have been carried out. Simulations of compressible turbulence are especially important in that they promote our understandings of wave features and wave-wave interactions in the solar wind turbulence. To our knowledge, most of them focus on the statistical properties of compressible modes regarded as components of MHD turbulence. Most relevant research and reviews - e.g., Cho et al. (2002), Cho and Lazarian (2003, 2005), Vestuto et al. (2003), Brunt and Mac Low (2004), Elmegreen and Scalo (2004), Zhou et al. (2004), Hnat et al. (2005), Kowal et al. (2007), Shivamoggi (2008), Kowal and Lazarian (2010), Tofflemire et al. (2011), and Brandenburg and Lazarian (2013) - have concentrated on spectral properties and/or anisotropy of different MHD wave modes. Among these, Hnat et al. (2005), Shivamoggi (2008), and Kowal and Lazarian (2010) focused on compressible modes and investigated their characteristics. There was also research investigating the correlations of quantities in turbulences as well as dominating modes in of MHD turbulences. Passot and Vazquez-Semadeni (2003) have found that which wave mode dominates the contribution to density fluctuations in turbulences is decided by $\theta_{\boldsymbol{k} \boldsymbol{B}}$ and Alfvénic Mach numbers $M_{\mathrm{A}}$. When $M_{\mathrm{A}}$ is low, the slow mode is more dominant, especially when $\theta_{\boldsymbol{k} \boldsymbol{B}}$ is close to $90^{\circ}$. Wisniewski et al. (2013) researched the ratio of energy of different modes as well as anisotropy. However, the local behaviors of MHD waves are seldom discussed.

Among these, it is worth highlighting the works of Cho et al. (2002) and Cho and Lazarian (2003, 2005). Those studies mainly covered the anisotropy of MHD waves turbulence spectra, but also extended their discussion to include the generation of fast/slow waves in ambient Alfvénic fluctuations. It was shown that fast/slow waves can be generated in pure Alfvén wave background (explicitly reported by Cho and Lazarian, 2005), but these articles did not include direct identifications of the generated MHD waves. Instead, only global, statistical parameters were extracted using a "projection" method. In order to "decompose" wave modes, complex amplitudes $\delta \boldsymbol{v}(\boldsymbol{k})$ were projected on eigenvectors determined by the background magnetic field $\boldsymbol{B}_{0}$ and wave vector $\boldsymbol{k}$. The Alfvén mode has velocity oscillations in the $\boldsymbol{B}_{0} \times \boldsymbol{k}$ direction, while the fast mode oscillates quasi-perpendicularly and slow mode the in quasi-parallel direction, both in the plane in which both $\boldsymbol{B}_{0}$ and $\boldsymbol{k}$ lie.

In this article we intend to identify slow wave trains from our simulations of MHD turbulences and describing their evolution and possible interaction with other types of MHD waves. Our goal differs from previous studies in that we provide direct and descriptive examples of slow waves, focusing

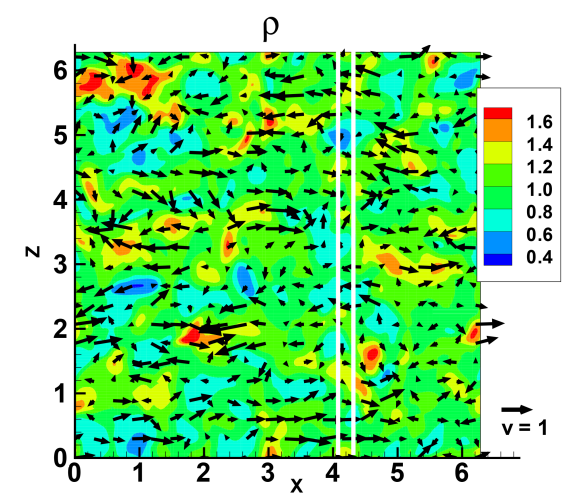

Figure 1. Density distribution (color) and in-plane velocities (arrows) in the plane $y=3.14$ at $t=0.71$. As a reference, an arrow is drawn to denote a velocity of 1 dimensionless unit. Between the white lines is the slice used in analyses of the slow wave case 1.

on local behaviors and deformation of the associated wave trains. Our criteria are insensitive to $\theta_{\boldsymbol{k} \boldsymbol{B}}$ and more versatile than the correlation between $\delta \rho$ and $\delta B_{\|}$.

In Sect. 2 we introduce our numerical model. In Sect. 3 we give a brief prescription of the identification of MHD waves and verify our new methods. In Sect. 4 we present our slow wave cases and compare them with the case of Y13. Finally, in Sect. 5 we summarize the results and present some discussions.

\section{Model description}

In this section, we briefly introduce the codes that we used in our compressible turbulence simulation. We have conducted an MHD simulation with codes employing a splittingbased finite-volume scheme (Feng et al., 2011; Zhang et al., 2011; Yang et al., 2013), where the magnetic field is computed with the constrained transport (CT) algorithm (Tóth, 2000 ) and the fluid part with a Gudonov-type central scheme (Ziegler, 2004; Fuchs et al., 2009). The codes are based on the PARAMESH package (MacNeice et al., 2000) and provide compressible solutions. (See Fig. 1.) The model is defined in a three-dimensional rectangular coordinate system, with $256 \times 256 \times 256$ grid points along the $x, y$, and $z$ directions, respectively. The corresponding computational region ranges from 0 to $2 \pi$ in each direction. The model describes an ideal MHD system whose adiabatic index $\gamma=5 / 3$, without explicit kinetic or magnetic viscosity terms.

All data and analyses presented in this article make use of a dimensionless unit system to simplify and clarify the illustration of the method. However, in order to interpret physical significances, a set of conversion factors should be set. In the solar wind at $1 \mathrm{AU}$, the typical Alfvén speed is about $100 \mathrm{~km} \mathrm{~s}^{-1}$, and we denote it as 2 dimensionless velocity units. Hence the speed unit is $v_{0}=50 \mathrm{~km} \mathrm{~s}^{-1}$. Typical solar wind contains about 5 protons per cubic centime- 
ter, and so we assign the mass density unit to be $\rho_{0}=8 \times$ $10^{-21} \mathrm{~kg} \mathrm{~m}^{-3}$. Their combination gives a unit of magnetic field $B_{0}=5.013 \mathrm{nT}$. Take a period of $t_{0}=20 \mathrm{~s}$ and the corresponding length is $L_{0}=1 \mathrm{Mm}$.

A simulation of decaying turbulences is performed. As initial conditions, we adopt random "broadband initial conditions" of Matthaeus et al. (1996). These initial conditions consist of a randomly superposed wave packet of velocity fluctuations $\boldsymbol{u}(\boldsymbol{k})$ with $|\boldsymbol{u}(\boldsymbol{k})|^{2} \propto 1 /\left(1+\left(k / k_{\text {knee }}\right)^{q}\right)$ in the $k$ range of $1<k<8$, where $k_{\text {knee }}$ is chosen to be 3 . The parameter $q$ decides the slope of the initial turbulence spectrum. For oscillations we set $\left\langle v_{x}^{2}\right\rangle=0.5$ and $\left\langle v_{y}^{2}\right\rangle=0.5$, as well as $\left\langle v_{x}\right\rangle=\left\langle v_{y}\right\rangle=0$. There are no oscillations of $v_{z}$ and $\boldsymbol{B}$. We uniformly set the mass density $\rho_{0}=1$, temperature $T_{0}=0.6$, and magnetic field strength $B_{z 0}=2, B_{x 0}=B_{y 0}=0$ in initial conditions, so that the initial Alfvén velocity $\left(v_{\mathrm{A}}=B_{0} / \sqrt{\rho_{0}}\right.$, in dimensionless unit system) is 2 , and the speed of sound $\left(c_{\mathrm{s}}=\sqrt{\gamma p_{0} / \rho_{0}}\right)$ is 1 . Hence, the plasma $\beta=0.3$ everywhere. These initial conditions intend to simulate the solar wind with in situ compressible turbulence and represent the local solar wind observations, yet they do not reflect radial expansion nor large-scale shear or stream interactions (Matthaeus et al., 1996). Hence, the simulation is limited to describing the local behaviors and cannot describe largescale phenomena, such as the radial evolution of solar wind turbulence.

We employ periodic boundary conditions on all six boundaries, considering the two following issues. Firstly, there is no rigid wall in the solar wind environment that our model simulates. Secondly, periodic boundary conditions correspond to wave modes propagating in an infinite space, so that the wave vector $\boldsymbol{k}$, which stems from Fourier analysis, makes sense.

\section{Wave mode diagnostics}

In this section we present our methods to identify slow wave trains in the simulation data. The identification is mainly accomplished with help of time-space slices along $\boldsymbol{B}_{0}$. We show that the slow wave trains exhibit a strong correlation between $\delta \rho$ and $\delta v_{\|}$, and such correlated structures propagate at a characteristic velocity. The theoretically predicted ratio $\left|\delta \rho / \delta v_{\|}\right|$changes little with varying $\theta_{\boldsymbol{k} \boldsymbol{B}}$, and thus the determination of the wave vectors can be bypassed. Though we fix on a single $\beta$ in the test case, these criteria are quite credible whenever $v_{\mathrm{A}}$ is quite above $c_{\mathrm{s}}$.

In Sect. 3.1, we describe the methods to identify slow waves, and we show the necessary features of other modes as well. The identification mainly involves the dispersion relation and polarity relations, where the angle $\theta_{\boldsymbol{k} \boldsymbol{B}}$ functions as a kernel parameter, yet the results are insensitive to it. In Sect. 3.2, we justify our method and check its robustness and applicability. We use a randomly generated wave packet to verify the correlation of $\delta \rho(t)$ and $\delta v_{\|}(t)$. The correla- tion only depends on the sign of $\cos \theta_{\boldsymbol{k} \boldsymbol{B}}$, regardless of the absolute value of $\cos \theta_{\boldsymbol{k} \boldsymbol{B}}$. The justification ensures that the method is reliable and available for analysis of time series.

\subsection{Identification and features of MHD waves}

Firstly, it is worth revisiting theoretical solutions of MHD waves, i.e., dispersion and polarity relations. These results are built on two hypotheses: (i) linearized oscillations, i.e., fluctuations, are small enough that higher order terms can be neglected, and (ii) there is only one monochromatic plane wave of one mode. With these hypotheses one can decompose a physical quantity $\psi$ into a constant "background" part and an oscillating part:

$\psi(\boldsymbol{x}, t)=\psi_{0}+\operatorname{Re}(\delta \hat{\psi} \exp (i(\boldsymbol{k} \cdot \boldsymbol{x}-\omega t))$,

where the background $\psi_{0}$ and the complex amplitude $\delta \hat{\psi}$ are constant. When only the oscillating part is discussed we will simply use $\delta \hat{\psi}$ instead.

Single-fluid MHD equations can only support three modes of waves: fast, slow, and Alfvén mode. If the speed of sound $c_{\mathrm{s}}$, Alfvén speed $v_{\mathrm{A}}$, and $\theta_{\boldsymbol{k} \boldsymbol{B}}$ are given, the phase velocities of fast and slow modes are determined according to

$$
\begin{aligned}
& V_{\mathrm{p}, \pm}\left(\theta_{\boldsymbol{k} \boldsymbol{B}}\right)= \\
& \sqrt{\frac{c_{\mathrm{s}}^{2}+v_{\mathrm{A}}^{2} \pm \sqrt{\left(c_{\mathrm{s}}^{2}+v_{\mathrm{A}}^{2}\right)^{2}-4 c_{\mathrm{s}}^{2} v_{\mathrm{A}}^{2} \cos ^{2} \theta_{\boldsymbol{k} \boldsymbol{B}}}}{2}},
\end{aligned}
$$

where the plus sign is for fast waves and minus for slow waves. The dispersion relation simply reads $\omega=V_{\mathrm{p}} k$.

For fast and slow mode, polarity relations in the form given by, for example, Olbers and Richter (1973) and Marsch (1986) are presented here (with some symbols altered and unit system changed so that the permeability of free space $\mu_{0}$ is 1$)$ :

$$
\begin{aligned}
& \delta \hat{\rho}=\epsilon \rho_{0}, \\
& \delta \hat{\boldsymbol{v}}=\epsilon \cdot \frac{V_{\mathrm{p}}}{V_{\mathrm{p}}^{2}-v_{\mathrm{A}}^{2} \cos ^{2} \theta_{\boldsymbol{k} \boldsymbol{B}}}\left(V_{\mathrm{p}}^{2} \hat{\boldsymbol{k}}-v_{\mathrm{A}}^{2} \cos \theta_{\boldsymbol{k} \boldsymbol{B}} \hat{\boldsymbol{B}}\right), \\
& \delta \hat{\boldsymbol{B}}=\epsilon \cdot \frac{V_{\mathrm{p}}^{2} v_{\mathrm{A}}}{V_{\mathrm{p}}^{2}-v_{\mathrm{A}}^{2} \cos ^{2} \theta_{\boldsymbol{k} \boldsymbol{B}}} \cdot\left(\hat{\boldsymbol{B}}-\cos \theta_{\boldsymbol{k} \boldsymbol{B}} \hat{\boldsymbol{k}}\right) \sqrt{\rho_{0}},
\end{aligned}
$$

where $\epsilon$ is the relative density fluctuation amplitude. Here it is plausible to omit the expression of $\delta \hat{p}_{\text {th }}$ from the solutions, since it is merely a passive quantity directly related to $\delta \hat{\rho}$ (e.g., Zank and Matthaeus, 1993). From the form, it is worth noting that both $\delta \boldsymbol{v}$ and $\delta \boldsymbol{B}$ lie in the plane defined by $\boldsymbol{B}_{0}$ and $\boldsymbol{k}$ (e.g., take $\boldsymbol{B}_{0}=\left(0,0, B_{0}\right)$ and $\boldsymbol{k}=\left(k_{x}, 0, k_{z}\right)$, and the fluctuations will lie in the $x-z$ plane). In that plane the parallel and perpendicular directions can be defined relative to $\boldsymbol{B}_{0}$. From Eq. (3b) the parallel and perpendicular components of 
(a) Wave phase speeds along $B_{0}$

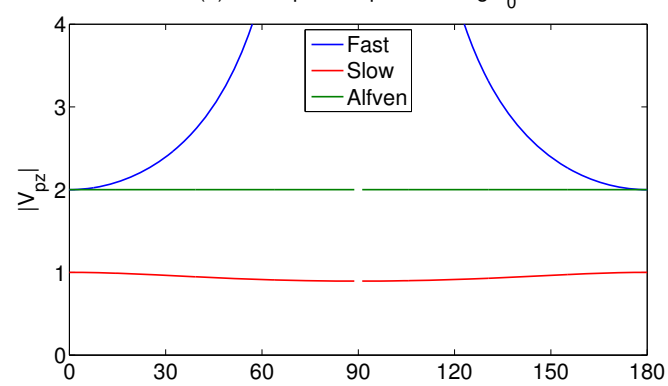

(b) Compression due to fast and slow waves

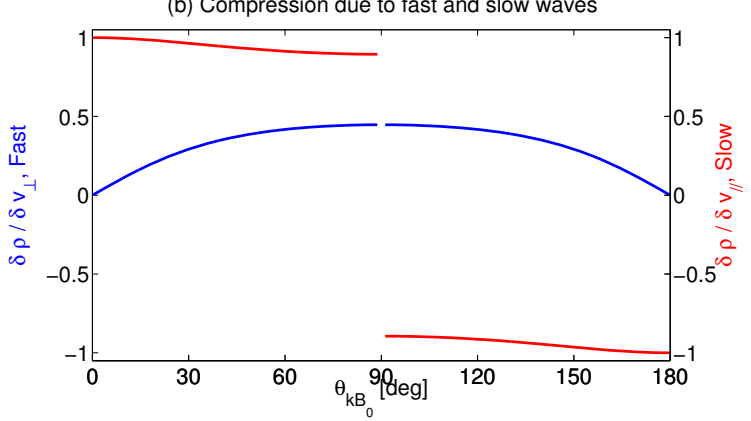

Figure 2. Theoretically predicted features of MHD waves. (a) Magnitudes of wave phase speed along $z$ direction $\left|V_{\mathrm{p} z}\right|$ versus angle $\theta_{\boldsymbol{k} \boldsymbol{B}}$. (b) Compression due to fast and slow waves versus different $\theta_{\boldsymbol{k} \boldsymbol{B}}$, with vertical axis representing $\delta \hat{\rho} / \delta \hat{v}_{\|}$for slow mode (red), and $\delta \hat{\rho} / \delta \hat{v}_{\perp}$ for fast mode (blue). Both horizontal axes represent $\theta_{\boldsymbol{k} \boldsymbol{B}}$ in degrees. Data are computed with $v_{\mathrm{A}}=2, c_{\mathrm{s}}=1$, and $\rho_{0}=1$.

$\delta \boldsymbol{v}$ are

$$
\begin{aligned}
& \delta \hat{v}_{\|}=\epsilon \cdot \frac{V_{\mathrm{p}}}{V_{\mathrm{p}}^{2}-\cos ^{2} \theta_{\boldsymbol{k} \boldsymbol{B}} v_{\mathrm{A}}^{2}}\left(V_{\mathrm{p}}^{2}-v_{\mathrm{A}}^{2}\right) \cos \theta_{\boldsymbol{k} \boldsymbol{B}}, \\
& \delta \hat{v}_{\perp}=\epsilon \cdot \frac{V_{\mathrm{p}}}{V_{\mathrm{p}}^{2}-\cos ^{2} \theta_{\boldsymbol{k} \boldsymbol{B}} v_{\mathrm{A}}^{2}} \cdot V_{\mathrm{p}}^{2} \sin \theta_{\boldsymbol{k} \boldsymbol{B}} .
\end{aligned}
$$

For an Alfvén wave, the dispersion relation reads

$V_{\mathrm{pA}}= \pm v_{\mathrm{A}} \cos \theta_{\boldsymbol{k} \boldsymbol{B}}$

where the plus sign corresponds to the case $k_{\|}>0$, and vice versa for the minus sign. The Alfvén wave has $\delta \hat{\rho}=\delta \hat{p}=0$. Its velocity and magnetic field oscillate in $\boldsymbol{k} \times \boldsymbol{B}_{0}$ direction, with

$\delta \hat{\boldsymbol{v}}=\mp \frac{\delta \hat{\boldsymbol{B}}}{\sqrt{\rho_{0}}}$,

with the minus sign for $k_{\|}>0$ and vice versa for $k_{\|}<0$.

Let the phase velocity of the wave be $V_{\mathrm{p}}$ and the propagation velocity of wave phase along $\boldsymbol{B}_{0}$ be $V_{\mathrm{p} z}$. Then, since $V=\omega / k$,

$V_{\mathrm{p} z}=\frac{\omega}{k_{z}}=\frac{\omega}{k \cos \theta_{\boldsymbol{k} \boldsymbol{B}}}=\frac{V_{\mathrm{p}}}{\cos \theta_{\boldsymbol{k} \boldsymbol{B}}}$.
Polarity of velocity and magnetic field oscillations
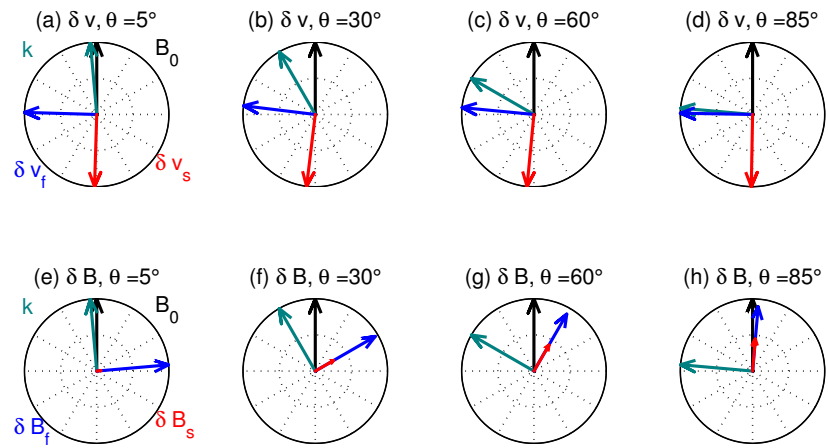

Figure 3. Polarity relations when $v_{\mathrm{A}}=2, c_{\mathrm{S}}=1$ and $\rho_{0}=1$. (a) to (d) show directions of velocity oscillations of fast and slow modes with different $\theta_{\boldsymbol{k} \boldsymbol{B}}$. (e) to (h) show directions of magnetic field oscillations of fast and slow modes. In each panel, the arrow length of $\delta \boldsymbol{B}$ is scaled proportional to $\delta \boldsymbol{v}$.

Since $V_{\mathrm{p} z}$ depends only on $\theta_{\boldsymbol{k} \boldsymbol{B}}$ instead of $\omega$, Eq. (7) also applies for wave packets with all components sharing the same $\theta_{\boldsymbol{k} \boldsymbol{B}}$. For fast, slow, and Alfvén modes we plot $V_{\mathrm{p} z}$ versus $\theta_{\boldsymbol{k} \boldsymbol{B}}$ in Fig. 2a. Along $\boldsymbol{B}_{0}$, Alfvén mode travels at a speed of 2 , independent of $\theta_{\boldsymbol{k} \boldsymbol{B}}$. For fast mode, $\left|V_{\mathrm{p} z}\right|$ is greater than or equal to 2 , more approximated to 2 at smaller $\theta_{\boldsymbol{k} \boldsymbol{B}}$. For slow mode, $\left|V_{\mathrm{p} z}\right|$ does not vary much from 1 . As a result, slowmode structures stand out due to the distinguishing velocity along $\boldsymbol{B}_{0}$, and the analysis of phase-propagating speed $\left|V_{\mathrm{p} z}\right|$ still works for general wave packets.

Next we analyze characteristic velocity oscillations of fast and slow waves. Since the wave energy density can be calculated as $\bar{\rho}\left\langle(\delta \boldsymbol{v})^{2}\right\rangle$, the amplitudes of $\delta \boldsymbol{v}$ can represent wave energy. In our case $v_{\mathrm{A}}=2$ and $c_{\mathrm{s}}=1$, and from Eqs. (4a) and (4b) the inequality $\left|\delta v_{\perp} / \delta v_{\|}\right|<0.12$ is valid, i.e., the oscillations of slow waves keep within $7^{\circ}$ (i.e., $\arctan 0.12$ ) relative to $B_{0}$ (see Fig. 3). Oscillations in parallel direction thus contribute at least $98 \%$ to the wave energy (because this percentage $\rho_{0} \delta v_{\|}^{2} /\left(\rho_{0} \delta v^{2}\right)$ can be written as $1 /\left(1+\delta v_{\perp}^{2} / \delta v_{\|}^{2}\right)$, which is greater than $\left.1 /\left(1+0.12^{2}\right) \approx 0.986\right)$. For fast mode, one can infer in the same fashion that the perpendicular direction contains almost all of the wave energy. Therefore, it is suitable to assign the parallel direction as the characteristic direction of velocity oscillations of slow mode, and the perpendicular one for the fast mode.

From Eqs. (3a), (4a) and (4b), one can derive that

$$
\begin{aligned}
\frac{\delta \hat{\rho}}{\delta \hat{v}_{\|}} & =\frac{\rho_{0} \cdot\left(V^{2}-\cos ^{2} \theta_{\boldsymbol{k} \boldsymbol{B}} v_{\mathrm{A}}^{2}\right)}{V \cdot\left(V^{2}-v_{\mathrm{A}}^{2}\right)} \cos \theta_{\boldsymbol{k} \boldsymbol{B}}, \\
\frac{\delta \hat{\rho}}{\delta \hat{v}_{\perp}} & =\frac{\rho_{0} \cdot\left(V^{2}-\cos ^{2} \theta_{\boldsymbol{k} \boldsymbol{B}} v_{\mathrm{A}}^{2}\right)}{V^{3} \sin \theta_{\boldsymbol{k} \boldsymbol{B}}} .
\end{aligned}
$$

Considering the characteristic velocity oscillation above, we take the former equation for the analysis of slow wave and the latter of fast wave. Figure $2 \mathrm{~b}$ clearly presents $\delta \hat{\rho} / \delta \hat{v}_{\perp}$ 
of fast mode and $\delta \hat{\rho} / \delta \hat{v}_{\|}$of slow mode. For slow mode, the polarity of $\delta \hat{\rho} / \delta \hat{v}_{\|}$depends on the signs of $k_{\|}$, and the ratio is not sensitive to $\theta_{\boldsymbol{k} \boldsymbol{B}}$ in each branch. In our case with $v_{\mathrm{A}}>c_{\mathrm{s}}$, maximal compression per unit "characteristic oscillation" occurs when $\theta_{\boldsymbol{k} \boldsymbol{B}}=0$ or $180^{\circ}$, where $\left|\delta \hat{\rho} / \delta \hat{v}_{\|}\right|=$ $\rho_{0} /\left|V\left(\theta_{\boldsymbol{k} \boldsymbol{B}}=0\right)\right|=\rho_{0} / c_{\mathrm{s}}$. The minimum occurs as $\theta_{\boldsymbol{k} \boldsymbol{B}} \rightarrow$ $90^{\circ}$, with $\left|\delta \hat{\rho} / \delta \hat{v}_{\|}\right| \rightarrow v_{\mathrm{A}} \rho_{0} /\left(c_{\mathrm{s}} \sqrt{v_{\mathrm{A}}^{2}+c_{\mathrm{S}}^{2}}\right)$. The ratio of the minimum to the maximum of $\left|\delta \hat{\rho} / \delta \hat{v}_{\|}\right|$is $v_{\mathrm{A}} / \sqrt{v_{\mathrm{A}}^{2}+c_{\mathrm{S}}^{2}}$ (in our case $\approx 0.894$ ). If $v_{\mathrm{A}}$ is much larger than $c_{\mathrm{s}}$, it is safe to assume

$$
\left|\delta \hat{\rho} / \delta \hat{v}_{\|}\right| \approx \frac{\rho_{0}}{c_{\mathrm{s}}} .
$$

For fast mode, when $\theta_{\boldsymbol{k} \boldsymbol{B}}$ is near 0 or $180^{\circ}$, there is no obvious compression, but when $\theta_{\boldsymbol{k} \boldsymbol{B}}$ is close to $90^{\circ}$, the ratio (Eq. 8b) approaches $\rho_{0} / \sqrt{v_{\mathrm{A}}^{2}+c_{\mathrm{S}}^{2}}$.

As above, the diagnosis of slow wave utilizes two criteria: wave phase speed along $\boldsymbol{B}_{0}$ and $\delta \rho / \delta v_{\|}$. The criteria work well in cases with $v_{\mathrm{A}}>c_{\mathrm{s}}$, yet better when the ratio $v_{\mathrm{A}} / c_{\mathrm{S}}$ is greater (i.e., with smaller $\beta$ ), where all the approximations applied here will be slightly more precise. In order to employ them in data analysis, we must derive a relation between $\delta \rho(t)$ and $\delta v_{\|}(t)$. In other words, we should further justify that

$$
\frac{\delta \rho(t)}{\delta v_{\|}(t)} \approx \pm \frac{\rho_{0}}{c_{\mathrm{S}}}
$$

also holds well. The plus sign is for wave packets consisting of slow wave with $k_{\|}>0$, and the minus sign for $k_{\|}<0$.

We also compute

$$
\begin{aligned}
\sigma_{\mathrm{c}}(\boldsymbol{x}, t) & =\frac{2\langle\delta \boldsymbol{v} \cdot \delta \boldsymbol{b}\rangle}{\langle\delta \boldsymbol{v} \cdot \delta \boldsymbol{v}\rangle+\langle\delta \boldsymbol{b} \cdot \delta \boldsymbol{b}\rangle}, \\
C_{p}(\boldsymbol{x}, t) & =\frac{\left\langle(\delta \rho)^{2}\right\rangle}{\rho_{0}^{2}} \frac{B_{0}^{2}}{\langle\delta \boldsymbol{B} \cdot \delta \boldsymbol{B}\rangle} .
\end{aligned}
$$

The quantity $\delta \boldsymbol{b}=\delta \boldsymbol{B} / \sqrt{\rho_{0}}$ denotes fluctuations of the magnetic fields, normalized to have velocity units. Gary and Winske (1992) pointed out that $\sigma_{\mathrm{c}}$ and $C$ distinguish wave modes sharply: in our case, slow waves should have $C_{p} \gg 1$ and negative $\sigma_{\mathrm{c}}$, Alfvén waves and "small-angled" fast waves should have $C_{p} \approx 0$ and $\sigma_{\mathrm{c}}$ near to -1 , and "large-angled" fast waves should have $C_{p}<1$ and $\sigma_{\mathrm{c}}$ between -1 and 0 (see Table 1, from Table 1a of Gary and Winske, 1992). These conclusions are for cases where $\boldsymbol{k} \cdot \boldsymbol{B}_{0}>0$. For cases where $\boldsymbol{k} \cdot \boldsymbol{B}_{0}<0, \sigma_{\mathrm{c}}$ will change signs, and $C_{p}$ will remain unchanged. The computation of the two parameters is identical to that of Y13 (cf. Y13's Eqs. 1 and 2).

In summary, MHD waves can be categorized into three types: (i) slow waves, (ii) Alfvén waves and quasi-parallel propagating fast waves (AW-like), and (iii) oblique and quasi-perpendicular propagating fast waves (fast-like). With $v_{\mathrm{A}}=2, c_{\mathrm{S}}=1$ and $\rho_{0}=1$ (typical for $\beta<1$ ), their behaviors can be listed and compared in Table 1. For completeness, the non-propagating entropy mode is also supplied. This mode has inhomogeneity of density and temperature, but keeps pressure, velocity and magnetic field strength all uniform. Since quasi-parallel phase-propagating slow waves show little $\delta \boldsymbol{B}$, it may be problematic to analyze correlations between $\delta \rho$ and $\delta B_{\|}$in such situations. However, since in the solar wind compressible fluctuations have larger $k_{\perp}$ than $k_{\|}$(Chen et al., 2012), such quasi-parallel cases may be neglected.

\subsection{Justification of methods}

The criteria described in Sect. 3.1 are based on the hypothesis that only a monochromatic wave of a given mode is involved. This is hardly the case in reality, where turbulent fluctuations have a wide range of frequencies and/or wave vectors, and different kinds of modes may be superposed.

To check the performances of the criteria in complicated cases, we have constructed a slow-mode wave packet to investigate the correlation of $\delta \rho$ and $\delta v_{\|}$. The packet consists of $N$ wave modes, i.e., for $\delta \rho$ and $\delta v_{\|}$

$$
\begin{gathered}
\delta v_{\|}(\boldsymbol{x}, t)=\sum_{i=1}^{N} \hat{v}_{\| i} \exp \left(\mathrm{i}\left(\boldsymbol{k}_{i} \cdot \boldsymbol{x}-\omega t\right)\right), \\
\delta \rho(\boldsymbol{x}, t)=\sum_{i=1}^{N} \hat{\rho}_{i} \exp \left(\mathrm{i}\left(\boldsymbol{k}_{i} \cdot \boldsymbol{x}-\omega t\right)\right) .
\end{gathered}
$$

For each mode $i$, we generate complex amplitude $\hat{v}_{\| i}$ with random moduli and initial phase. Each wave mode has a random $\boldsymbol{k}$, whose magnitude and direction are randomly decided with only one restriction $\theta_{\boldsymbol{k} \boldsymbol{B}}<89^{\circ}$. This is intent to guarantee that $k_{\|}$keeps the same sign in the wave packet. The corresponding $\omega$ is determined by $\boldsymbol{k}$ from dispersion relation. Hence we can calculate $\hat{\rho}_{i}$ from $\hat{v}_{\| i}$ with Eq. (8a), which describes the polarity relation between $\hat{\rho}$ and $\hat{v}_{\|}$.

To test Eq. (10), we computed time profiles at the point $\boldsymbol{x}=(0,0,0)$ of $\delta v_{\|}$and $\delta \rho$. The parameters are taken as in our initial condition: $v_{\mathrm{A}}=2, c_{\mathrm{s}}=1$, and $\rho_{0}=1$, so we expect the ratio to be about 1 . For each of $N=80$ modes, amplitudes of $\delta v_{\| i}$ are randomly selected in the range from 0 to 1 , initial phase from 0 to $2 \pi$, azimuthal angle of $\boldsymbol{k}_{\perp}$ (relative to $\boldsymbol{B}_{0}$ ) from 0 to $2 \pi$, and $|k|$ from 0 to 2 in order to implement the random superposition of wave modes and extend the range of frequencies. The plots of $\delta v_{\|}$and $\delta \rho$ almost overlap in Fig. 4 , which implies the ratio $\approx 1$. In this way the method is justified.

If other modes coexist, the correlation between $\delta \rho$ and $\delta v_{\|}$ may be (or not) destroyed, depending on the type of the coexisting wave. Fast and Alfvén waves do not have obvious $\delta v_{\|}$. As a result, observed $\delta v_{\|}$is contributed by slow waves. The correlation is possibly affected when the coexisting mode is either "large-angled" fast wave or slow wave propagating in the opposite direction. At the same time, however, whenever only Alfvén waves and/or parallel propagating fast waves coexist, the correlation will remain intact. To analyze mixed 
Table 1. Features of MHD waves.

\begin{tabular}{lllll}
\hline & (i) Slow & (ii) AW-like & (iii) Fast-like & (iv) Entropy \\
\hline$\delta \boldsymbol{v}$ & $\|$ & $\perp$ & $\perp$ & 0 \\
$\delta \rho$ & $\approx \pm 1$ & $\sim 0$ & $\sim 1 / 2$ & arbitrary \\
& $\left(\right.$ to $\left.\delta v_{\|}\right)$ & $\left(\right.$to $\left.\delta v_{\perp}\right)$ & $\left(\right.$ to $\left.\delta v_{\perp}\right)$ & \\
$|\delta \boldsymbol{B}|$ & $\sim 0(\boldsymbol{k}$ quasi $\|)$ & $\sim 1$ & $\sim 1$ & 0 \\
& $\sim 1 / 2($ quasi $\perp)$ & & & \\
$\left|V_{\mathrm{p} z}\right|$ & $\approx 1$ & $\sim 2$ & $>2$ & 0 \\
$C_{p}$ & $\gg 1$ & $\sim 0$ & $<1$ & $\infty$ \\
$\sigma_{\mathrm{c}}$ & $<0\left(k_{\|}>0\right)$ & $\sim-1$ & -1 to 0 & $\mathrm{n} / \mathrm{a}$ \\
& $>0\left(k_{\|}<0\right)$ & $\sim 1$ & 0 to 1 & \\
\hline
\end{tabular}

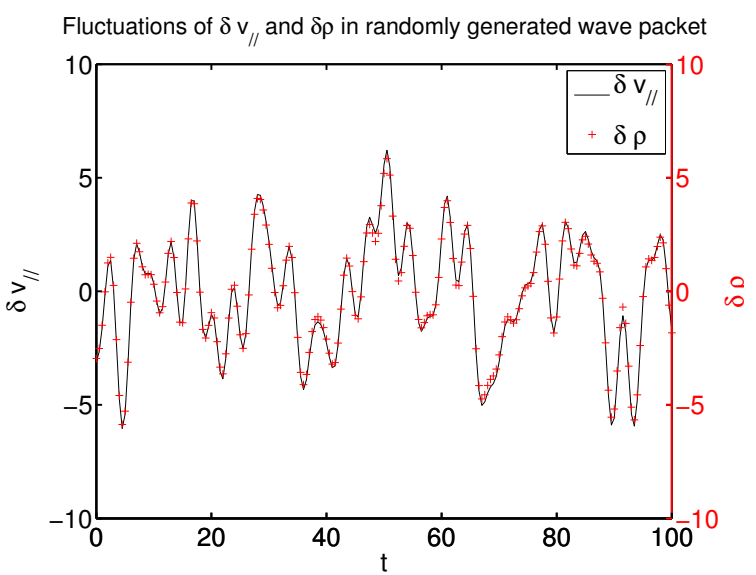

Figure 4. Time profiles of $\delta v_{\|}(t)$ and $\delta \rho(t)$ of a composed wave packet containing slow waves with random amplitudes, random wave vectors $\left(k_{\|}>0\right)$, and random initial phase. The vertical axis is for oscillating quantities, and the horizontal axis represents time $t$.

wave forms, linear spectral models (e.g., Klein et al., 2012) may be used along the methods presented above to separate different modes with help from eigenmode solutions.

\section{Results}

In this section we analyze the data from compressible simulations with the methods above and diagnose possible slow wave cases, four of which are presented with one in detail and the others in brief.

The data are extracted in time-space slices with coordinates $x$ and $y$ fixed, so that the slices lie in the $z$ direction. The primary characteristics of the cases are listed in the Table 2 . Propagating speeds are computed by fitting the track of phase in the $(z, t)$ diagram. Averaged values and oscillations are evaluated with "wave points" (defined in the next paragraph).

For case 1 (see the white lines in Fig. 1), profiles of characteristic quantities are plotted in Fig. 5. Red squares in panel a track the slow wave train. The points tracked are
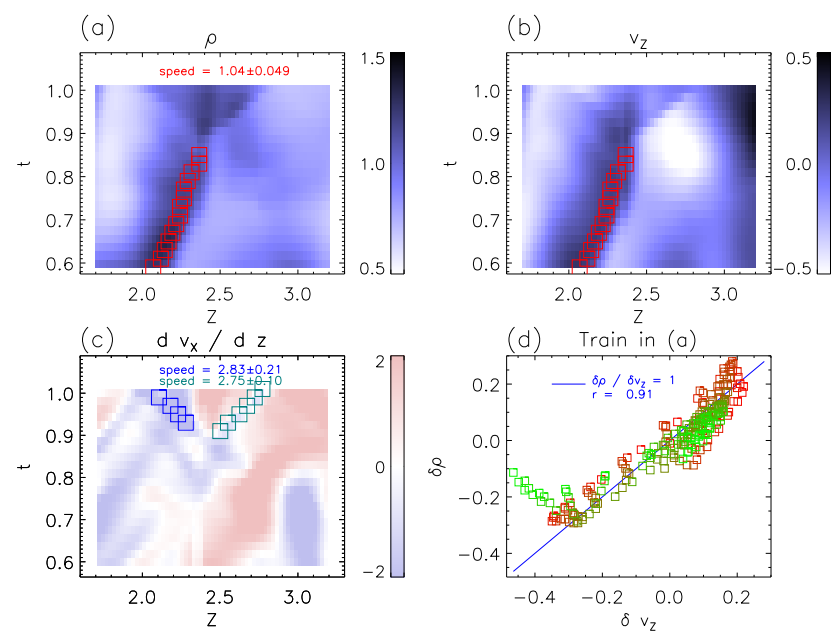

Figure 5. Temporal evolution of quantities near propagating region of slow wave case 1. (a) Temporal profiles of density $\rho$, with slow wave train displayed. (b) Temporal profiles of $z$ component of velocity $v_{z}$, with the same $(z, t)$ points as shown in (a). (c) Temporal profiles of $\mathrm{d} v_{x} / \mathrm{d} z$, with two secondary wave trains displayed. (d) Distribution of $\delta v_{z}$ and $\delta \rho$ at points near the slow wave train displayed in (a). In (a) to (c) the horizontal axis represents coordinate $z$ in the slice. The vertical axis marks the times of corresponding profiles. The colors plot the corresponding physical quantities. In (d) the blue line plots $\delta \rho / \delta v_{z}=1$ as a reference; colors of markers show their time, with red ones earlier and green ones later. The fluctuation values are obtained by subtraction of the average of the quantities at these points.

maxima of density $\rho$. Panel b plots a $z-t$ profile of $v_{z}$ with the same tracked points marked. Since slow waves usually appear shortly in turbulences, their widths are confined so that we can analyze the region near to the marked points. For each time $t$ with the wave peak marked at $z=z_{\mathrm{p}}(t)$, we take the points where $z_{\mathrm{p}}-0.2 \leq z<z_{\mathrm{p}}+0.2$ and define them as "wave points". An estimation of background quantities can be obtained from the average of all wave points through the time span, giving $\overline{\boldsymbol{B}} \approx(-0.1,-0.5,2.0)$ and $\overline{\boldsymbol{v}} \approx(-0.4,-0.3,0.04)$ in $(x, y, z)$ coordinates. The angle between $\overline{\boldsymbol{B}}$ and $z$ axis is only $14^{\circ}$. In our qualitative analy- 
Table 2. Overview of the cases analyzed.

\begin{tabular}{lccllrlrrr}
\hline Case & $x$ & $y$ & $z$ & $t$ & $\begin{array}{r}\text { Propagating } \\
\text { speed along } z\end{array}$ & $\bar{\rho}$ & $\left\langle\left(\delta v_{z}\right)^{2}\right\rangle$ & $\begin{array}{r}\bar{E}_{\text {Slow }} \\
\text { Correlation } \\
r\left(\delta v_{z}, \delta \rho\right)\end{array}$ \\
\hline 1 & 4.1 & 3.14 & 2.0 to 2.4 & 0.55 to 0.85 & $1.04 \pm 0.049$ & 1.06 & 0.18 & 0.032 & 0.91 \\
$2 \mathrm{~L}$ & 1.6 & 3.14 & 4.2 to 3.4 & 0.65 to 1.37 & $-1.04 \pm 0.014$ & 0.91 & 0.31 & 0.087 & -0.86 \\
$2 \mathrm{R}$ & 1.6 & 3.14 & 5.2 to 3.8 & 0.65 to 1.85 & $-1.11 \pm 0.011$ & 0.95 & 0.29 & 0.078 & -0.78 \\
3 & 5.0 & 3.14 & 3.1 to 2.7 & 0.49 to 0.71 & $-0.84 \pm 0.057$ & 1.18 & 0.17 & 0.034 & -0.91 \\
4 & 3.1 & 3.14 & 5.1 to 5.2 & 0.37 to 0.55 & $0.92 \pm 0.081$ & 1.10 & 0.20 & 0.049 & 0.94 \\
\hline
\end{tabular}

sis it is reasonable to approximate the $z$ direction as the parallel direction. The background velocity $\bar{v}_{z}$ is not large enough to force the subtraction of this velocity from the wave speed along the $z$ slice. Meanwhile, $\bar{\rho}$ and $B_{z}$ change little from the initial condition, and thus the initial values are still valid as background values. The root-mean-square value of $\delta v_{z}$ is about 0.2 , and $\delta \rho \approx 0.2$. This confirms to the polarity relations, and the root-mean-square values show that the wave train is linear in amplitude.

Panels a and $\mathrm{b}$ exhibit characteristic properties of a slow wave train: (1) an obvious density change ( $20 \%)$, (2) typical propagating velocity $(1.04 \pm 0.049)$ around $c_{\mathrm{s}}=1$, and (3) good correlation between fluctuations of $\rho$ and $v_{\|}$(correlation coefficient $=0.91)$.

Equation (10) serves as another important criterion for slow-mode waves. To check against it, we have analyzed the correlation between $\delta v_{z}$ and $\delta \rho$ (Fig. 5d). As a reference, a blue line is plotted corresponding to $\delta \rho / \delta v_{\|}=1$. The points gather around the blue line, highlighting a typical strong correlation for slow waves. From the distribution the averages and oscillations are also calculated, so the averaged wave energy density can be calculated as $\bar{E}_{\mathrm{w}}=\bar{\rho}\left\langle\left(\delta v_{z}\right)^{2}\right\rangle$, and is listed in Table 2.

To explain Y13's observation, we further compute some features employed in Y13's analysis. We take points where $0 \leq z<4.2$ on the slice at $t=0.71$. The running smooth window used to compute $C_{p}$ and $\sigma_{\mathrm{c}}$ has a width of 0.5 , chosen as about a typical scale of the wave (see Fig. 5a). The results are presented in Fig. 6, where panels $a$ and $b$ are plotted without smoothing. Panel $\mathrm{b}$ shows the correlation of $v_{x}$ and $B_{x}$ as the result of searching for possible Alfvénic structures. Since the $x$ direction can be interpreted as a perpendicular direction, the Alfvén mode or small-angled fast mode both have $\delta v_{\perp} \approx \mp \delta B_{\perp} / \sqrt{\bar{\rho}}$ (in the case of Alfvén waves, the equal sign holds strictly). In the segment marked with blue vertical lines in Fig. 6b, a negative correlation between $B_{x}$ and $v_{x}$ can be seen, and the amplitudes of their change are almost equal. In the same plot, the segment $z \leq 1$ shows almost overlapping profiles of $B_{x}$ and $v_{x}$. The two evidences may suggest an Alfvénic background. In this way, an Alfvén or small-angled fast-mode background is expected. For comparison with Gary and Winske (1992), panels c and d give parameters of the wave train. The wave points show $C \gg 1$

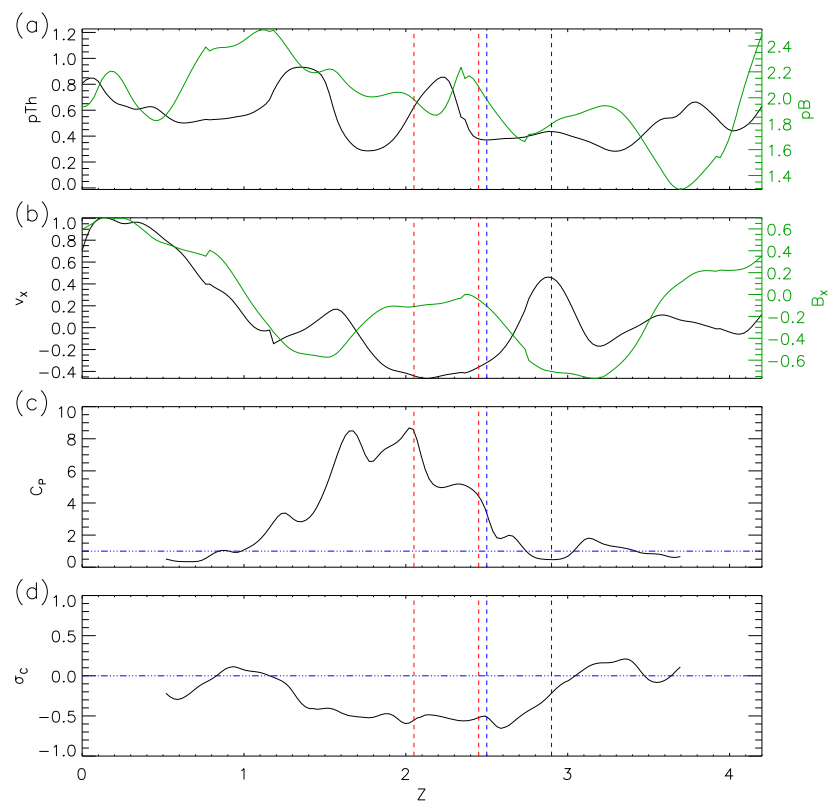

Figure 6. Features of points near density maximum in case 1, at $t=0.71$. (a) Instant values of thermal pressure $p_{\mathrm{th}}=\rho T$ (black) and magnetic pressure $p_{B}=B^{2} / 2$ (green). (b) Instant values of perpendicular components of velocity $v_{x}$ (black) and magnetic field $B_{x}$ (green). (c) Compressibility $C$ computed with Eq. (11b). (d) Dimensionless cross-helicity $\sigma_{\mathrm{c}}$ computed with Eq. (11a). Horizontal axes represent the $z$ coordinate along the slice, which is taken at $x=4.10, y=3.14$, and $0 \leq z<4.2$. In (a) and (b), both vertical axes are adjusted so that they share the same scale. Red lines mark "wave points", and the vertical blue lines mark a region of negative correlation of $v_{x}$ and $B_{x}$.

and $\sigma_{\mathrm{c}} \approx-0.5$, which agrees well with the criteria. Therefore the slow-mode wave train is again identified.

Thus we identified a slow wave train embedded in Alfvénic-like structures, which explains Y13's observation. However, the wave train does not propagate forever. This is also described in Fig. 5a and b. At $t=0.87$, the density peak in panel a becomes hardly recognizable and starts to blur. At the same moment, the $v_{z}$ structure in panel $\mathrm{b}$ does not propagate any further. The slow wave train seems to be deformed. In Fig. 5a, a fan-like structure appears, hinting at a possible generation of at least two waves and propagation in oppo- 

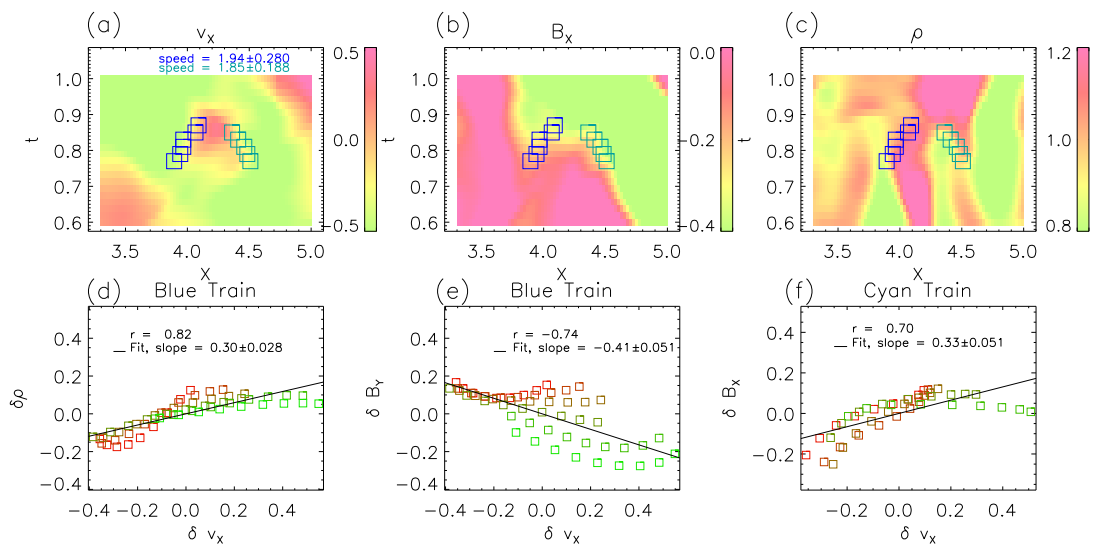

Figure 7. Temporal evolution of quantities along the $x$ slice in case 1, taken to intersect the $z$ slice in Fig. 5. (a) Temporal profiles of $v_{x}$, with two wave trains displayed. (b) Temporal profiles of $B_{x}$. (c) Temporal profiles of $\rho$. (d) Distribution of $\delta \rho$ and $\delta v_{x}$ at points near the blue (left) wave train. (e) Distribution of $\delta B_{y}$ (profile not plotted) and $\delta v_{x}$ at points near the blue wave train. (f) Distribution of $\delta B_{x}$ and $\delta v_{x}$ at points near the cyan (right) wave train. In (d) to (f), the points are selected as ones within 0.1 unit length to the tracked points. Redder markers stand for earlier time points and greener for later.

site directions. To check them, we plot $\mathrm{d} v_{x} / \mathrm{d} z$ in panel $\mathrm{c}$ and marked in both wave trains in blue and cyan squares at local minima of the profile, respectively. These waves have the speed of fast waves, and moreover they cause density change. They are possibly "large-angled" fast waves.

To understand what happens to the wave train when it deforms, we take another slice along the $x$ axis at the same time interval and with $z=2.3$ and $y=3.14$, so that it passes the site where the slow wave train begins to deform. We plot the corresponding profiles in Fig. 7, with maxima of $\left|\partial v_{x} / \partial x\right|$ tracked, and we have them marked in panels a to $c$. The series of the maxima can be regarded as wave trains. The blue wave train exhibits some density fluctuations (see panel c), which are much smaller than in slow mode. To understand its wave mode, we perform two fits (see panels $\mathrm{d}$ and e). Its behaviors resemble fast waves with $\theta_{\boldsymbol{k} \boldsymbol{B}} \sim 40^{\circ}$ (cf. Figs. 2a and 3). For the cyan wave train, the oscillations of $B_{x}$ and $v_{x}$ are similar (see panel b). A scatterplot of $B_{x}$ and $v_{x}$ near the cyan train is provided in panel $\mathrm{f}$, displaying a positive correlation. The two trains are not slow waves, the blue one behaves "fastlike", and the cyan one behaves somehow "Alfvén-like". It is notable that they meet at $z \approx 4.1$ and $t=0.87$, the very point where the slow wave train deforms.

Hence, the deforming of the slow wave train in case 1 can be described in such a scenario: a slow wave train interacts with at least two non-slow counterparts (one of them probably being "large-angled" fast waves), and deforms into at least two non-slow ones.

For other cases, only overviews of the slow wave trains are presented. In case 2 (Fig. 8), two slow wave trains propagate together. However, no clear wave form between the peaks is revealed. In contrast to case 1, the two wave trains remain quite a long time, especially for the wave train at larger $z$ (case $2 \mathrm{R}$ ). Moreover, waves in case 2 propagate "down-
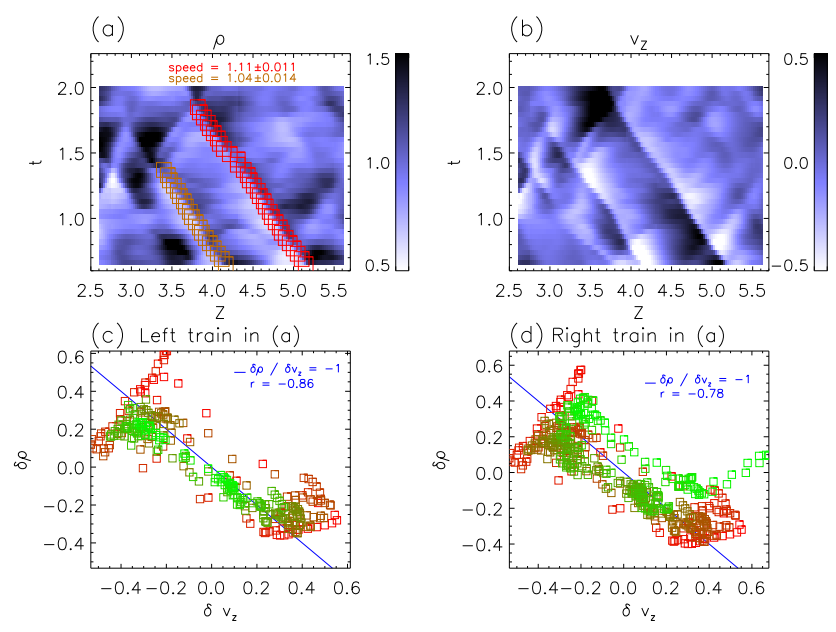

Figure 8. Features of slow wave case 2. In (a), the orange markers show case $2 \mathrm{~L}$ and the red ones case $2 \mathrm{R}$.

wards" to smaller $z$, while the counterpart in case 1 propagates "upwards" to larger $z$. Accordingly, $\delta \rho$ and $\delta v_{\|}$show negative correlation in case 2. Case 3 is a rather short case, whose profiles are shown in Fig. 9. Because we take fewer points along the wave train, the correlation coefficient $r$ appears larger than that of cases 1 and 2. Before the slow wave train is formed, a fast wave train exists (see the cyan markers in panel c). At $t \sim 0.47$, it seems to bifurcate, with most of its energy being transferred into the slow-mode train. A minor part continues propagating as fast or Alfvén mode, which is barely detectable in panel c after $t=0.47$. This scenario resembles the one discussed by Kudoh and Shibata (1999). After $t=0.67$, the slow wave train appears to excite another sharp wave train (panel c). However, the slow wave train does not fully deform. Case 4 is also a rather short case with pro- 

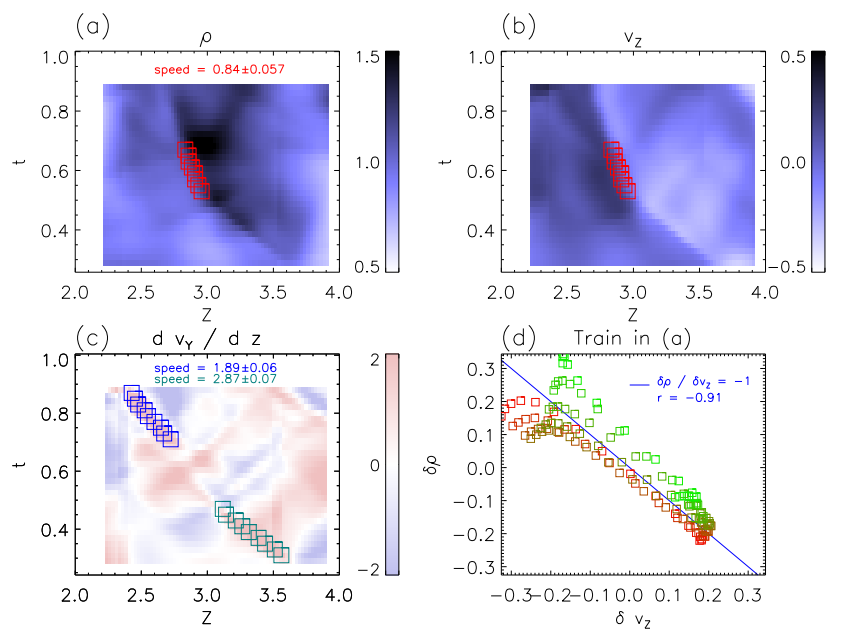

Figure 9. Features of slow wave case 3, in the same format as Fig. 5.

files shown in Fig. 10. The excited wave is so weak that it can barely be detected (see panel c). However, the energy of the deformed slow wave train may be contributed to the complicated velocity structure shown at the top of Fig. 10b. In all cases, the slow wave trains seem to be destroyed as they interact with other modes at the same point. After the destruction, new wave trains might be formed and shape "fan-like" structures shown in time-space slices of density. Such structures are best shown in case 1, but still detectable in cases 2 to 4 (cf. Figs. 8a, 9a, and 10a).

\section{Summary and discussions}

This article establishes criteria to identify slow wave trains in MHD simulation data, and presents four cases of such slow wave trains. Several of their physical properties are extracted. The main results are summarized here.

1. The identification of slow wave trains is achieved by studying data along a slice approximately aligned to the background magnetic field. Typical slow wave behaviors include $\left|V_{\mathrm{p} z}\right| \approx \pm c_{\mathrm{s}}$ and quite strong correlation of $\delta \rho$ and $\delta v_{\|}$, with their ratio $\sim \pm \rho_{0} / c_{\mathrm{s}}$. They are consistent with the derivation from dispersion and polarity relations of linear MHD oscillations. These properties are dominated only by the sign of $\cos \theta_{\boldsymbol{k}} \boldsymbol{B}$ and insensitive to its absolute value, allowing us to bypass the calculation of wave vector $\boldsymbol{k}$. The criteria are theoretically reliable if $v_{\mathrm{A}}$ is well above $c_{\mathrm{s}}$, and they have passed a test with randomly generated wave packets.

2. Four cases of linear slow wave train are analyzed. They comply well with the criteria. For case 1, the parameters $\sigma_{\mathrm{c}}$ and $C_{p}$ are computed, and they support the identification.
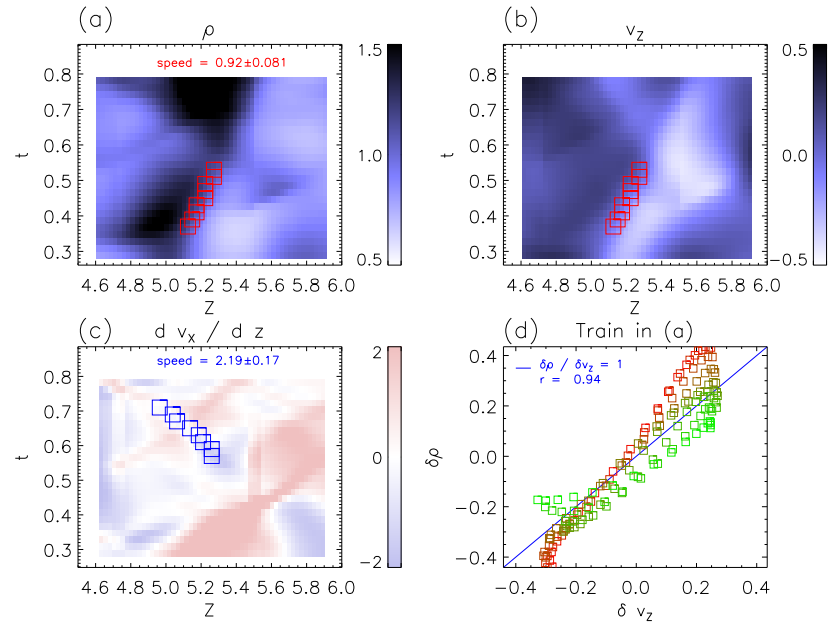

Figure 10. Features of slow wave case 4 , in the same format as Fig. 5. In (a) and (b), tracked points are maxima of $|\partial \rho / \partial z|$.

3. Near the slow wave train of case 1, an Alfvénic structure exists. This explains Y13's observation as a local slow wave train generated in an Alfvénic environment, except that the existence of a pressure-balanced structure cannot be clearly confirmed.

4. Such slow wave trains are inclined to dissolve. They deform suddenly, probably when they interact with other waves, and their density structures will expand so that they dissolve like a fan in time-space slice plots. The boundary of the fan propagates at a typical speed of fast wave, and the plasma there shares properties of the fast wave or Alfvén wave. This is clearly shown by case 1, but in other cases the fan-like density structures are also detectable (though barely).

We have computed the wave energy density of the four wave trains, and found that the wave energy densities are about 0.03 to 0.04 except in case 2 . All points in the computational region at $t=0.71$ give $\rho_{0}\left\langle(\delta \boldsymbol{v})^{2}\right\rangle \approx 0.39$. Since the zones of compressible modes occupy a small portion of the computational region, and energy densities of slow wave trains are much smaller than the background fluctuations, it is reasonable to roughly estimate the energy density of Alfvén mode as 0.4. Since all four cases appear at time spans near $t=0.71$, this background energy density applies to all cases in a rough estimation. Therefore, the typical wave energy density of slow wave trains amounts about 0.1 in all fluctuations. (For case 2, this proportion might be 0.2.)

There are certain limitations in this work. Firstly, this research is limited within MHD descriptions. Kinetic magnetosonic waves behave differently from MHD eigenmodes (Klein et al., 2012). Therefore, kinetic effects (not included in our MHD investigation) may limit applications of the reported MHD methods to solar wind observations. Slow mode waves generally suffer more damping than Alfvén waves do. 
Damping rates of slow modes vary with $\theta_{\boldsymbol{k} \boldsymbol{B}}$, with the quasiperpendicular ones less damped (Barnes, 1966; Schekochihin et al., 2009). In the solar wind, the compressive fluctuations are found to be anisotropic with $k_{\perp}$ larger than $k_{\|}$, based on the structure function analysis (Chen et al., 2012). This explains why slow-mode fluctuations exist in the solar wind even though they suffer some damping. Nevertheless, our MHD model is limited in that it does not cover slow wave damping. Secondly, this work mainly focuses on phenomenal features of slow waves. However, their genesis has not yet been discussed, because our initial condition is a rather complex one and we need a method to trace the development of wave trains. With such a method, it would be possible to perform further investigations, such as analysis of fan structures in density profile, mechanisms of wave interactions, and relations between such wave trains and structures (e.g., discontinuities or intermittencies), Thirdly, though in principle our numerical simulation is able to produce a time series of 3$\mathrm{D}$ data, we are forced to select only the essential information for the analysis because of the formidable computational cost when high-resolution $(x, y, z, t)$ data are produced and analyzed. Local analysis of such wave trains, which utilizes information about the three-dimensional and temporal evolution at local space-time points, may be conducted in future research. The analyses presented in this article employ slices and thus provide a workable compromise. Nevertheless, this research has to bypass some aspects such as determination of wave vectors, which may be essential for further analysis.

Acknowledgements. This work is supported by NSFC grants under contracts 41231069, 41174148, 41222032, 41274172, 41031066, and 41304133. The PARAMESH software used in this work was developed at the NASA Goddard Space Flight Center and Drexel University as a part of NASA's HPCC and ESTO/CT projects and with support from grant NNG04GP79G from the NASA/AISR project. J.-S. He is also supported by a foundation for the Author of National Excellent Doctoral Dissertation of P.R. China (FANEDD) under contract no. 201128. The authors would like to thank the referees for suggestions that led to improvement in this article.

Topical Editor L. Blomberg thanks K. Klein and the one anonymous referee for their help in evaluating this paper.

\section{References}

Barnes, A.: Collisionless Damping of Hydromagnetic Waves, Phys. Fluids, 9, 1483, doi:10.1063/1.1761882, 1966.

Belcher, J. W. and Davis, L.: Large-amplitude Alfvén waves in the interplanetary medium, J. Geophys. Res., 76, 3534-3563, doi:10.1029/JA076i016p03534, 1971.

Brandenburg, A. and Lazarian, A.: Astrophysical Hydromagnetic Turbulence, Space Sci. Rev., 178, 163-200, doi:10.1007/s11214013-0009-3, 2013.

Bruno, R. and Carbone, V.: The Solar Wind as a Turbulence Laboratory, Living Reviews in Solar Physics, 2, 4, doi:10.12942/lrsp2005-4, 2005.
Brunt, C. and Mac Low, M.: Modification of projected velocity power spectra by density inhomogeneities in compressible supersonic turbulence, Astrophys. J., 604, 196-212, doi:10.1086/381648, 2004.

Chen, C. H. K., Mallet, A., Schekochihin, A. A., Horbury, T. S., Wicks, R. T., and Bale, S. D.: Three-dimensional structure of solar wind turbulence, Astrophys. J., 758, 120, doi:10.1088/0004637X/758/2/120, 2012.

Cho, J. and Lazarian, A.: Compressible magnetohydrodynamic turbulence: mode coupling, scaling relations, anisotropy, viscosity-damped regime and astrophysical implications, Mon. Not. R. Astron. Soc., 345, 325-339, doi:10.1046/j.13658711.2003.06941.x, 2003.

Cho, J. and Lazarian, A.: Generation of compressible modes in MHD turbulence, Theor. Comp. Fluid Dyn., 19, 127-157, doi:10.1007/s00162-004-0157-x, 2005.

Cho, J., Lazarian, A., and Vishniac, E. T.: Simulations of Magnetohydrodynamic Turbulence in a Strongly Magnetized Medium, Astrophys. J., 564, 291, doi:10.1086/324186, 2002.

Elmegreen, B. and Scalo, J.: Interstellar turbulence I: Observations and processes, Annu. Rev. Astron. Astr. , 42, 211-273, doi:10.1146/annurev.astro.41.011802.094859, 2004.

Feng, X., Zhang, S., Xiang, C., Yang, L., Jiang, C., and Wu, S. T.: A Hybrid Solar Wind Model of the CESE+HLL Method with a Yin-Yang Overset Grid and an AMR Grid, Astrophys. J., 734, 50, doi:10.1088/0004-637X/734/1/50, 2011.

Fuchs, F. G., Mishra, S., and Risebro, N. H.: Splitting based finite volume schemes for ideal MHD equations, J. Comput. Phys., 228, 641-660, doi:10.1016/j.jcp.2008.09.027, 2009.

Gary, S. P. and Winske, D.: Correlation function ratios and the identification of space plasma instabilities, J. Geophys. Res.-Space, 97, 3103-3111, doi:10.1029/91JA02752, 1992.

Goldstein, M., Roberts, D., and Matthaeus, W.: MAgnetohydrodynamic turbulence in the solar-wind, Annu. Rev. Astron. Astr. , 33, 283-325, doi:10.1146/annurev.astro.33.1.283, 1995.

He, J., Marsch, E., Tu, C., Yao, S., and Tian, H.: Possible Evidence of Alfvén-cyclotron Waves in the Angle Distribution of Magnetic Helicity of Solar Wind Turbulence, Astrophys. J., 731, 85, doi:10.1088/0004-637X/731/2/85, 2011.

He, J., Tu, C., Marsch, E., and Yao, S.: Do Oblique Alfvén/Ioncyclotron or Fast-mode/Whistler Waves Dominate the Dissipation of Solar Wind Turbulence near the Proton Inertial Length?, Astrophys. J. Lett., 745, L8, doi:10.1088/2041-8205/745/1/L8, 2012.

Hnat, B., Chapman, S., and Rowlands, G.: Compressibility in solar wind plasma turbulence, Phys. Rev. Lett., 94, 204502, doi:10.1103/PhysRevLett.94.204502, 2005.

Howes, G. G., Bale, S. D., Klein, K. G., Chen, C. H. K., Salem, C. S., and TenBarge, J. M.: The slow-mode nature of compressible wave power in solar wind turbulence, Astrophys. J., 753, L19, doi:10.1088/2041-8205/753/1/L19, 2012.

Kellogg, P. J. and Horbury, T. S.: Rapid density fluctuations in the solar wind, Ann. Geophys., 23, 3765-3773, doi:10.5194/angeo23-3765-2005, 2005.

Klein, K., Howes, G., TenBarge, J. M., Bale, S., Chen, C., and Salem, C.: Using synthetic spacecraft data to interpret compressible fluctuations in solar wind turbulence, Astrophys. J., 755, 159-174, doi:10.1088/0004-637X/755/2/159, 2012. 
Kowal, G. and Lazarian, A.: Velocity field of compressible magnetohydrodynamic turbulence: wavelet decomposition and mode scalings, Astrophys. J., 720, 742-756, 2010.

Kowal, G., Lazarian, A., and Beresnyak, A.: Density fluctuations in MHD turbulence: Spectra, intermittency, and topology, Astrophys. J., 658, 423-445, doi:10.1086/511515, 2007.

Kudoh, T. and Shibata, K.: Alfvén wave model of spicules and coronal heating, Astrophys. J., 514, 493-505, 1999.

MacNeice, P., Olson, K. M., Mobarry, C., de Fainchtein, R., and Packer, C.: PARAMESH: A parallel adaptive mesh refinement community toolkit, Comput. Phys. Commun., 126, 330-354, doi:10.1016/S0010-4655(99)00501-9, 2000.

Marsch, E.: Acceleration potential and angular momentum of undamped MHD-waves in stellar winds, Astron. Astrophys., 164, 77-85, 1986.

Marsch, E.: Kinetic Physics of the Solar Corona and Solar Wind, Living Reviews in Solar Physics, 3, 1, doi:10.12942/lrsp-2006$1,2006$.

Matthaeus, W. H., Ghosh, S., Oughton, S., and Roberts, D. A.: Anisotropic three-dimensional MHD turbulence, J. Geophys. Res., 101, 7619-7629, doi:10.1029/95JA03830, 1996.

Olbers, D. J. and Richter, A. K.: Wave-trains in the solar wind, Astrophys. Space Sci., 20, 373-389, doi:10.1007/BF00642209, 1973.

Passot, T. and Vazquez-Semadeni, E.: The correlation between magnetic pressure and density in compressible MHD turbulence, Astron. Astrophys., 398, 845-855, doi:10.1051/00046361:20021665, 2003.

Podesta, J. J. and Gary, S. P.: Magnetic Helicity Spectrum of Solar Wind Fluctuations as a Function of the Angle with Respect to the Local Mean Magnetic Field, Astrophys. J., 734, 15, doi:10.1088/0004-637X/734/1/15, 2011.

Salem, C. S., Howes, G. G., Sundkvist, D., Bale, S. D., Chaston, C. C., Chen, C. H. K., and Mozer, F. S.: Identification of Kinetic Alfvén Wave Turbulence in the Solar Wind, Astrophys. J., 745, L9, doi:10.1088/2041-8205/745/1/L9, 2012.

Schekochihin, A., Cowley, S., Dorland, W., Hammett, G., Howes, G., Quataert, E., and Tatsuno, T.: Astrophysical gyrokinetics: kinetic and fluid turbulent cascades in magnetized weakly collisional plasmas, Astrophys. J. Supply S., 182, 310, doi:10.1088/0067-0049/182/1/310, 2009.

Shivamoggi, B. K.: Magnetohydrodynamic turbulence: Generalized formulation and extension to compressible cases, Ann. Phys.New York, 323, 1295-1303, doi:10.1016/j.aop.2008.02.008, 2008.
Tofflemire, B. M., Burkhart, B., and Lazarian, A.: Interstellar sonic and alfvenic mach numbers and the tsallis distribution, Astrophys. J., 736, 60, doi:10.1088/0004-637X/736/1/60, 2011.

Tóth, G.: The $\nabla \cdot B=0$ Constraint in Shock-Capturing Magnetohydrodynamics Codes, J. Comput. Phys., 161, 605-652, doi:10.1006/jcph.2000.6519, 2000.

Tu, C. and Marsch, E.: MHD structures, waves and turbulence in the solar-wind - observations and theories, Space. Sci. Rev., 73, 1-210, doi:10.1007/BF00748891, 1995.

Vestuto, J., Ostriker, E., and Stone, J.: Spectral properties of compressible magnetohydrodynamic turbulence from numerical simulations, Astrophys. J., 590, 858-873, doi:10.1086/375021, 2003.

Wang, X., He, J., Tu, C., Marsch, E., Zhang, L., and Chao, J.-K.: Large-amplitude Alfvén Wave in Interplanetary Space: The Wind Spacecraft Observations, Astrophys. J., 746, 147, doi:10.1088/0004-637X/746/2/147, 2012.

Wisniewski, M., Kissmann, R., and Spanier, F.: Turbulence evolution in MHD plasmas, J. Plasma Phys., 79, 597-612, doi:10.1017/S0022377813000147, 2013.

Yang, L., He, J., Peter, H., Tu, C., Chen, W., Zhang, L., Marsch, E., Wang, L., Feng, X., and Yan, L.: Injection of plasma into the nascent solar wind via reconnection driven by supergranular advection, Astrophys. J., 770, 6, doi:10.1088/0004-637X/770/1/6, 2013.

Yao, S., He, J., Tu, C., Wang, L., and Marsch, E.: Smallscale pressure-balanced structures driven by oblique slow mode waves measured in the solar wind, Astrophys. J., 774, 59, doi:10.1088/0004-637X/774/1/59, 2013.

Zank, G. P. and Matthaeus, W.: Nearly incompressible fluids. II: Magnetohydrodynamics, turbulence, and waves, Phys. Fluids AFluid, 5, 257-273, 1993.

Zhang, S.-H., Feng, X.-S., Wang, Y., and Yang, L.-P.: Magnetic Reconnection Under Solar Coronal Conditions with the 2.5D AMR Resistive MHD Model, Chinese Phys. Lett., 28, 089601, doi:10.1088/0256-307X/28/8/089601, 2011.

Zhou, Y., Matthaeus, W., and Dmitruk, P.: Colloquium: Magnetohydrodynamic turbulence and time scales in astrophysical and space plasmas, Rev. Mod. Phys., 76, 1015-1035, doi:10.1103/RevModPhys.76.1015, 2004.

Ziegler, U.: A central-constrained transport scheme for ideal magnetohydrodynamics, J. Comput. Phys., 196, 393-416, doi:10.1016/j.jcp.2003.11.003, 2004. 